\title{
THE NEUROLOGICAL ADAPTATION OF ICD-10 (ICD-10 NA)
}

\author{
JENNY J. VAN DRIMMELEN-KRABBE *
}

The systematic categorization of disorders, provided by the World Health Organization in the "International Statistical Classification of Diseases, Injuries and Causes of Death" (ICD) is an essential tool for epidemiological and clinical research, for the indexing of national and international statistics of morbidity and mortality, to monitor health services and to formulate public health policy. The Tenth Revision was adopted in 1990, and the classification was published in 1992 '.

As the ICD is primarily an instrument for the reporting of national and international statistics of morbidity and mortality, it is therefore subject to the constraint of providing for each category or subcategory one unique code number which shows its place within the classification. An important characteristic of the structure of the ICD is that it can be adapted to the different types of users: the "core" of 3 and 4 character codes can be further subdivided for use by specialties, where more detailed diagnoses are needed, but they can also be contracted or condensed with less detail and broader groups, e.g. for use in primary care. In development are several adaptations of ICD-10 to specialties, one of which is the neurological adaptation, at present in print. Already for ICD-9 a neurological adaptation has been prepared and published for trial purposes ${ }^{2}$. It was received with interest by the scientific community and other users. In view of this experience WHO has undertaken the preparation of a neurological adaptation to the ICD-10 as well.

The neurological adaptation aims

1. to provide one unique code for each recognized neurological disorder;

2. to encourage making detailed diagnoses using as many codes as necessary;

3. to provide a standard recording system for neurological disorders, available in several languages;

4. to encourage national and international epidemiological research for support of programmes of prevention and control.

The need to be internationally acceptable implies an extensive process of consultation with members states and their professional organizations, so that as many viewpoints as are practicable and compatible are represented in the classification. Thus for the development of the neurological adaptation of ICD-10 support has been sought through and provided by many leading international organizations in the field of neuroscience (see Table 1).

Table 2 shows an example of a category which can be found in the ICD-10NA: G43 Migraine. The titles with 3 and 4 characters are exactly the same as in the core ICD-10. The additional categories with 5 and 6 characters have been designed for use within the neurological field. This additional subcategorization has been developed in close collaboration with among others the International Headache Society ${ }^{3}$.

Another development is the production of "fascicles" that deal with a selected group of disorders within ICD-10 and bundle together all information that is needed in classifying this group of disorders. This is useful since the divisional structure of ICD-10 into 21 different chapters has, as a consequence, that codes for etiology and codes for the manifestation of certain illnesses may be found in different chapters. Moreover, these fascicles will contain definitions and diagnostic criteria for this selected group of disorders. The fascicles are produced by leading international organizations in this specific field, in cooperation with WHO. Table 3 gives an overview of the fascicles that are at present in preparation. They will become available in the course of 1995 and 1996. More fascicles may be prepared in the future for the other groups of disorders.

*Medical Officer, Division of Mental Health, World Health Organization (WHO), Geneva.

Dr Jenny J. van Drimmelen-Krabbe - Division of Mental Health, WHO - CH-1211 Geneva 27 - Switzerland. 
Table 1. Non-governmental organizations supporting the development of ICD-10 NA.

American Academy of Neurology

American Neurological Association

American Sleep Disorders Assocation

European Federation of Neurology

International Bureau for Epilepsy

International Brain Research Organization

International Child Neurology Association

International Cerebral Palsy Society
International Federation of Multiple Sclerosis Societies

International Headache Society

International League against Epilepsy

International Movement Disorder Society

International Society of Neuropathology

International Stroke Society

World Federation of Mental Health

World Federation of Neurology

World Federation of Neurosurgical Societies

Table 2. Example of a category in the ICD-10 NA.

\section{G43 Migraine}

Use additional external cause code (Ch. XX), if desired, to identify drug, if drug-induced

Excludes: headache NOS (R51)

atypical facial pain (G50)

G43.0 Migraine without aura [common migaine]

G43.1 Migraine with aura [classical migraine]

G43.10 With typical aura

G43.11 With prolonged aura

G43.12 With acute onset aura

Use sixth character, if desired, to identify neurological symptoms:

G43.1 00 Hemianopic and other visual migraine

G43.1x1 Hemisensory migraine

G43.1 2 Migraine with aphasia

G43.1 $\times 3$ Basilar migraine

G43.1x4 Migraine aura (all types) without headache

G43.1×5 Farnilial hemiplegic migraine

G43.1x7 Multiple types of aura

G43.1 $\times 8$ Other specified migraine with aura

Table 3. Fascicles, linked to ICD-10, in preparation.

Cerebrovascular disorders

Epilepsy

Headaches

Hereditary ataxias

Mental retardation

Movement disorders
Multiple sclerosis

Neuromuscular disorders

Pain disorders

Psychogeriatry, including Dementia

Sleep disorders

\section{REFERENCES}

1. International Statistical Classification of Diseases and Related Health Problems. Tenth Revision. Vol I: Tabular list, 1992. Vol 2: Instruction Manual, 1993. Vol 3: Index, 1994. Geneva: WHO.

2. Application of the International Classification of Diseases to Neurology (ICD NA). Geneva: WHO, 1987.

3. Orgogozo J-M, Van Drimmelen JJ, Bradley WG, Sartorius $N$. The WHO view on Disease Classification and Diagnosis. Copenhagen: Proceedings of Headache Research Seminar, Nov 19-21, 1993. 